\title{
Enzymatic Competition: Modeling and Verification with Timed Hybrid Petri Nets
}

\author{
Sylvie Troncale*, Jean-Paul Comet** and Gilles Bernot** \\ * IBISC, University of Evry, 523 Places des Terrasses 91000 Evry, France \\ Email: sylvie.troncale@ibisc.fr \\ ${ }^{* *}$ Laboratoire I3S, UMR 6070 UNSA-CNRS, Algorithmes-Euclide-B, 2000, route \\ des Lucioles, B.P. 121, 06903 Sophia Antipolis CEDEX, France \\ Email:\{comet, bernot\}@unice.fr
}

\begin{abstract}
The formalism of Hybrid Functional Petri Nets (HFPN) has proved its convenience for simulating biological systems. The drawback of the noticeable expressiveness of HFPN is the difficulty to perform formal verifications of dynamical properties. In this article, we propose a model-checking procedure for Timed Hybrid Petri Nets (THPN), a sub-class of HFPN. This procedure is based on the translation of the THPN model and of the studied property into real-time automata. It is applied to model enzymatic competitions existing in amphibian metamorphosis.
\end{abstract}

Key words: Metamorphosis, verification, Timed hybrid Petri nets, model-checking

\section{Introduction}

Systems biology aims to the understanding of biological systems taking into account molecular processes, signal transduction, interactions between subcellular compartments... For this goal researchers are interested in modeling of biological systems. A lot of modeling frameworks focus on the simulation of biological systems: local evolution laws are given in a strong expressive way and simulations are used to make emerge some complex behavioral properties of the global system. For example, Hybrid Functional Petri Nets (HFPN) [7] allow the representation in a unified way of discrete and continuous entities (e.g. number of cells or molecular concentration) as well as discrete and continuous processes (e.g. mitosis is an example of discrete event although evolution of concentrations due to enzymatic reactions exemplifies a continuous one). The functional aspects of HFPN allow the definition of biological functions in terms of mathematical functions associated with processes, leading to a 
stronger expressiveness of this formalism. At the opposite, other approaches focus on the capability of checking whether the in silico model satisfies properties of the biological system (model checking) [9]: only a model which is coherent with the known biological behavioral properties is a relevant model.

In this paper, we propose to make a compromise between expressiveness and verification. We choose to restrict the expressiveness of HFPN by removing its functional aspects because they induce some implicit use of system states. For this obtained sub-class of HFPN, called Timed Hybrid Petri Nets (THPN) [3], we developed a model checking procedure based on the translation of the THPN model and of the studied property, into real time automata. Depending on whether there exist common executions between both automata, we conclude whether the THPN model satisfies or not the studied property. We apply our model checking procedure on a sub-network involved in the amphibian metamorphosis. This biological application, performed in collaboration with a team of developmental biologists, leads to new crucial biological questions.

The paper is organized as follows: Section 2 presents the studied biological system. Section 3 recalls major definitions and properties of THPN. Sections 4 and 5 present the conversion into real-time automata of the THPN model and of the property respectively. Finally, intuitions of the procedure of final decisions are given in Section 6 before we discuss our results in Section 7.

\section{Biological Context}

Most amphibians undergo numerous morphological changes at the tadpole stage, a biological process called metamorphosis. Amphibian metamorphosis can be divided into three periods. During premetamorphosis the feeding tadpole grows. During prometamorphosis hind limbs grow and differentiate. Finally, tail resorption characterizes the metamorphic climax. All metamorphosis-associated morphological changes are under the control of the thyroid hormone, denoted TH. It is relevant to distinguish two molecular forms of TH [4]. Thyroxine (TH4) corresponds to the major form secreted by the thyroid gland, it is an "inactive" form of TH and is considered a pro-hormone. Triiodothyronine (TH3) is the biologically active form but it is secreted in a smaller quantity [12].

The TH-dependent regulation at the origin of tail resorption requires a strong intra-cellular TH concentration [4]. This regulation is triggered at the climax of metamorphosis when plasmatic TH concentration is maximal. Intra-cellular TH bind their nuclear receptors, noted TR [13]. Complexes TH3/TR are transcription factors inducing at an early time, a set of early genes and at a later 
time, a set of late genes responsible for apoptosis.

The intra-cellular TH3 concentration depends on the plasmatic contribution, but it is also regulated by two enzymes, type 2 deiodinase, D2 [2] and type 3 deiodinase D3 [6]. Type 2 deiodinase synthesizes the active form of the hormone (TH3) from its inactive form (TH4) : D2 + TH4 $\rightarrow$ D2 + TH3. On the contrary, type 3 deiodinase inactivates the two hormone types TH3 and TH4 $: D 3+$ TH $4 \rightarrow D 3+r T H 3$ and D3 + TH3 $\rightarrow D 3+T 2$, where rTH3 and T2 are inactive. The gene expressing D3 is a direct-response gene of $\mathrm{TH}$ [6]. Expression of gene encoding D2 is also regulated by thyroid hormone, but in an indirect manner. D2 concentration then increases with the one of TH3, but response time is much longer (few days) [2].

In a previous work [11], a HFPN model representing kinetics of different factors which interfere in tail resorption was constructed. The main goal of such a modeling work was to lead to a better understanding of the biological system. In our case, the model was constructed with the goal of detailling our knowledge about specific functionalities of thyroid hormone in the tail. The initial question refers to the TH nature: can we talk about morphogen for thyroid hormone? Recall that a morphogen is a secreted molecule which has the property to induce different cellular types with different concentrations.

Nevertheless, model construction leads to expose new pertinent questions. Indeed, it is easy to remark that TH3 undergoes to two retroaction loops: a positive retroaction loop with D2 and a negative retroaction loop with D3. It is commonly thought that the intra-cellular concentration of TH3 becomes sufficient to induce tail resorption thanks to D2 action [2]. Nevertheless, the kinetics data ( $\mathrm{Km}$ and $\mathrm{V} \max$ ) given by Germain et al. in [5] show that D2 is 10 times less active than D3. We then ask us on D2 role. Is D2 sufficient to induce an strong enough concentration of TH3? To answer this question, we model in this paper competition existing between the two loops. Thus, the question to be asked can be informally exposed as follows: "At the climax of metamorphosis, when the concentration of $\mathrm{TH}$ is maximal, does always the negative loop overcome the positive loop ?" Our model-checking procedure allows to verify whether the model satisfies the previous biological property.

\section{$3 \quad$ Timed Hybrid Petri Nets}

The competitive regulation between D2 and D3 is modeled by a Timed Hybrid Petri Net. Evolution of a THPN can then be studied by constructing its evolution graph. Let us first recall the definition of THPN models and of evolution graphs [3]. 
Definition 1 A Timed Hybrid Petri Net is an 8-tuple $\left(\mathcal{P}, \mathcal{T}, \zeta\right.$, Pre, Post, $m_{0}$, delay, $\left.V\right)$ where:

- $\mathcal{P}$ and $\mathcal{T}$ are disjoint sets of places and transitions.

- $\zeta: \mathcal{P} \cup \mathcal{T} \rightarrow\{D, C\}$ called "hybrid function," indicates for every node whether it is a discrete node or a continuous one.

Let $T^{D}$ (resp. $\left.P^{D}\right)$ and $T^{C}$ (resp. $\left.P^{C}\right)$ be the sets of discrete and continuous transitions (resp. places).

- Pre $: \mathcal{P} \times \mathcal{T} \rightarrow \mathbb{Q}^{+} \cup \mathbb{N}$ is the input incidence application. If $T \in T^{D}$ then $\operatorname{Pre}(P, T) \in \mathbb{N}$ else Pre $(P, T) \in \mathbb{Q}^{+}$.

- Post $: \mathcal{T} \times \mathcal{P} \rightarrow \mathbb{Q}^{+} \cup \mathbb{N}$ is the output incidence application. If $T \in T^{D}$ then $\operatorname{Post}(T, P) \in \mathbb{N}$ else Post $(T, P) \in \mathbb{Q}^{+}$.

- $m_{0}: \mathcal{P} \rightarrow \mathbb{R}^{+} \cup \mathbb{N}$ is the initial marking. If $P \in P^{D}$ then $m_{0}(P) \in \mathbb{N}$ else $m_{0}(P) \in \mathbb{R}^{+}$.

- delay $: T^{D} \rightarrow \mathbb{Q}^{+}$is a timing associated with each discrete transition.

- $V: T^{C} \rightarrow \mathbb{Q}^{+}$represents the maximal firing speed associated with each continuous transition.

Biological example: THPN. Continuous places model biological entities which cannot be numbered (real value). Molecular concentrations of TH3, TH4, D2 and D3 are then modeled by continuous places (Figure 1). Initially, we considered no D2 and no D3 and we considered a factor 5 between the concentrations of TH4 and TH3 [2]. THPN transitions model discrete or continuous biological processes. Since D3 is a direct-response gene of TH3, its continuous induction by the hormone is then modeled by a continuous transition $\left(T_{2}\right)$. At the opposite, D2 is induced in an indirect manner, what leads to a delay between TH3 induction and D2 synthesis. This indirect induction is modeled by the discrete transition $T_{1}$. The continuous transition $T_{3}$ models D3 activity by removing TH3 and TH4 and the continuous transition $T_{4}$ models D2 action by synthesizing TH3 from TH4. Note that TH3, D2 and D3 are not consumed during respectively, enzyme induction and enzyme action, consequently a bidirectional arc is used.

\section{FIGURE 1}

Semantics intuition. Definition 1 gives a syntactic definition of THPN. So as to understand the dynamics of THPN, let us describe their semantics. A discrete transition $T$ is enabled if each place $P_{i}$ preceding $T$ satisfies $m\left(P_{i}\right) \geq \operatorname{Pre}\left(P_{i}, T\right)$, where $m\left(P_{i}\right)$ is the current marking of the place $P_{i}$ (Figure 1: $T_{1}$ is enabled if the place TH3 reaches the threshold 1$)$. If the discrete transition $T$ stays enabled during the time $\operatorname{delay}(T)$, it will be fired at the end of this delay. $\operatorname{Pre}\left(P_{i}, T\right)$ tokens are then removed from each place $P_{i}$ and $\operatorname{Post}\left(T, P_{j}\right)$ tokens are added to each place $P_{j}$ succeeding $T$ (Figure 1: firing of $T_{1}$ induces the production of one token in D2 and TH3, as well as the consumption of one token in TH3). A continuous transition $T$ is enabled if each 
place $P_{i}$ preceding $T$ satisfies $m\left(P_{i}\right)>0$ (Figure 1: $T_{3}$ is enabled if places TH3 and TH4 are not empty). A continuous transition is fired to its instantaneous firing speed $v(T)$ such that $0 \leq v(T) \leq V(T)$. $v(T)$ corresponds to the maximal speed a transition can fire according to the current marking. A flow of $\operatorname{Pre}\left(P_{i}, T\right) \times v(T)$ tokens are removed from each place $P_{i}$ preceding $T$ and a flow of $\operatorname{Post}\left(T, P_{j}\right) \times v(T)$ tokens are added to each place $P_{j}$ succeeding to $T$.

Evolution graph. The behavior of a THPN can be represented by an evolution graph (EG), represented by a Petri net [3]. Each place corresponds to an IB-state (invariant behavior state) and each transition is associated with an event (change of marking) whose occurrence produces a change of IB-state. Such a transition can only occur if an event belonging to one of the following types takes place: the marking of a continuous place becomes zero, a discrete transition fires or a discrete transition becomes enabled because of a change of continuous place marking.

Intuitively, a transition of the evolution graph (denoted $T^{E G}$ ) is labeled with the set of occurred events, with time of the event occurrence and with marking of all continuous places. IB-states are annotated by marking of all discrete transitions and by the vector of instantaneous speeds (see Figure 1). To construct such an evolution graph, a restriction is imposed to THPN [3]. Since the evolution graph represents a deterministic behavior, one has to solve conflicts which occur when the marking of a place is not sufficient to allow the different transitions to fire simultaneously. Generally, conflicts are solved by priority, by ranking involved transitions.

Biological example: EG. The place $T H 3$ (Figure 1) must fire three different transitions: $T_{1}, T_{2}$ and $T_{3}$. If the marking of $T H 3$ is not sufficient to allow these three firings, a conflict exists. Different resolution rules can be given: $T_{1}$ has priority then $T_{2}$ and $T_{3}, T_{2}$ has priority,... Figure 1 gives the evolution graph of the THPN model when the transition $T_{3}$ has priority. For pedagogical reasons, only one conflict resolution is handled. Nevertheless, our method handles all conflict resolutions. An evolution graph with several branches is then obtained, each branch corresponds to a conflict resolution.

\section{Event Clock Automaton of the THPN}

Each branch of the evolution graph gives an infinite timed execution, called a timed trace of the THPN. The set of timed traces of a THPN is represented by a real-time automaton. Thus, for studying a THPN, we have first to convert timed traces of the evolution graph into this real-time automaton, called Event Clock automaton [1]. After definition of an Event Clock automaton (ECA), our algorithm allowing the translation of an evolution graph into an ECA is 
illustrated on the evolution graph of the Figure 1.

A timed trace of a THPN informs us about the value of each variable (marking, speed,...) at some given times. Observations of THPN variables are called atoms. For example, the following atoms Fire $\left(T_{1}\right), m(T H 4) \geq 2.0$ and NoEvent mean that the discrete transition $T_{1}$ is fired, the marking of the place $T H 4$ is greater than 2.0 and no specific event occurred, respectively. The Event Clock automaton associated with the evolution graph models succession of atoms in the course of THPN execution. This ECA is timed thanks to the use of two clocks, the history clock $x_{\alpha}$ and the prophecy clock $y_{\alpha}$ [1], where $\alpha$ is an atom. The value of a history clock $x_{\alpha}$ is the time elapsed since the last occurrence of $\alpha$. The value of a prophecy clock $y_{\alpha}$ is the time to wait for the next occurrence of $\alpha$. For example, the clock constraint $y_{p} \leq 3$ means that $p$ will be true in at most 3 time units and $x_{q}=4$ means that $q$ was observed 4 time units ago.

Definition 2 An Event Clock automaton is a 6-tuple $A=\left(L, L_{0}, A t, \mathcal{C}, E, \mathcal{F}\right)$ where:

- $L$ is a finite set of locations, and $L_{0} \subseteq L$ is the subset of start locations.

- At is a set of atoms.

- $\mathcal{C}$ is a set of history or prophecy clocks.

- $E$ is a finite set of edges. An edge is a 4 -tuple $\left(l_{1}, \psi, l_{2}, w\right)$ where $l_{1} \in L$ is the source location, $l_{2} \in L$ is the target location, $\psi$ is a set of atoms describing the location $l_{1}$, and $w$ is a clock constraint.

- $\mathcal{F}=\left\{F_{1}, \ldots, F_{n}\right\}$, where $F_{i} \subseteq L$, is a set of sets of accepting locations.

Algorithm. Each transition of the evolution graph gives a location of the ECA, called $A_{M}$. Edges outgoing from these locations are labeled by atoms describing the evolution graph transitions: marking of each place, speed value of each continuous transition or the set of occurred events. Moreover, time is associated with transitions of the EG. Two successive transitions $\left(T_{1}^{E G}\right.$ and $T_{2}^{E G}$ ) labeled by times $t_{1}$ and $t_{2}$, respectively are then separated by $t_{2}-t_{1}=$ $\Delta t$. This delay labels ECA edges thanks to history clocks. The Event Clock automaton $A_{M}$ resulting from the evolution graph of Figure 1 is partially shown in Figure 2. The first transition of this EG gives the location $l_{0}$ in $A_{M}$. The edge outgoing from this location is labeled by the atoms: NoEvent, $m($ TH3) $=$ $1, v\left(T_{3}\right)=1, \ldots$

Each IB-state also gives a location in $A_{M}$. Atoms associated with IB-state stay true during all the time $\Delta t$. Execution then loops on these locations. Edges outgoing from these locations are labeled by these atoms. The first IB-state (Figure 2) gives the location $l_{1}$. One of the two edges outgoing from $l_{1}$ loops. They are labeled by the following atoms: $v\left(T_{3}\right)=1, v\left(T_{4}\right)=0, \ldots$

The start location is the location corresponding to the first transition of the 
evolution graph $\left(l_{0}\right)$. The set of sets of accepting locations is reduced to singletons containing only the locations corresponding to the last IB-states $\left(l_{8}\right)$.

FIGURE 2

\section{$5 \quad$ Study of a property}

Timed traces of a THPN integrate notions of real time. The studied property is then expressed into a real-time logic, we called Continuous Time Evolution Logic (CTEL). Definition 3 presents syntactic rules of a CTEL formula.

Definition 3 Following [8], a CTEL formula is composed of atoms, connectives $\neg, \vee, \wedge$, temporal operators Next $(\bigcirc)$, Previous $(\ominus)$, Until $(U)$, and Since (S), and of real-time operators: predicting and history operators ( $\triangleright$, $\triangleleft)$ :

$$
\varphi::=a\|\neg \varphi\| \bigcirc \varphi\|\ominus \varphi\| \varphi_{1} \wedge \varphi_{2}\left\|\varphi_{1} \vee \varphi_{2}\right\| \varphi_{1} U \varphi_{2}\left\|\varphi_{1} S \varphi_{2}\right\| \triangleleft_{\sim n} a \| \triangleright_{\sim n} a,
$$

where $a$ is an atom, $\sim \in\{=,<,>, \leq, \geq\}, \varphi, \varphi_{1}, \varphi_{2}$ are formulas and $n \in \mathbb{Q}^{+}$.

The studied property translates into a set of admissible behaviors of the system. Consequently, a property expressed in CTEL translates into a set of timed traces which can also be translated into an Event Clock automaton. If we study the property $f$, the Event Clock automaton $A_{\neg f}$ of the negation of $f$ is constructed. $A_{\neg f}$ represents the set of all timed traces of $\neg f$. We can then search whether there exists a trace common to $A_{M}$ and $A_{\neg f}$. If such a trace exists, we conclude that our THPN model does not satisfy $f$ since there exists a trace satisfying $\neg f$. Raskin and Schobbens proposed an algorithm to construct an Event Clock automaton from a property [8]. This algorithm (not detailed here) is complete, a timed trace satisfying $\neg f$ is then necessarily represented by the automaton.

Biological example. Our THPN model simulates competition existing between the two retroaction loops: the positive loop with D2 and the negative loop with D3. We ask us whether in all cases of conflict resolutions, the negative loop overcomes the positive loop. This property is translated into a CTEL formula $f: f \equiv \square \diamond(m(T H 3)=0)$ where $\diamond$ means eventually and $\square$ means globally. Both operators $\diamond$ and $\square$ correspond to the following abbreviations: let $\phi$ be a CTEL formula, then $\diamond \phi \equiv$ True $U \phi$ and $\square \phi \equiv \neg \diamond \neg \phi$.

The formula means that the concentration of TH3 finally reaches zero, what translates into the predominance of the negative loop. Figure 3 shows the 
Event Clock automaton of $\neg f \equiv \neg \square \diamond(m(T H 3)=0)$.

\section{FIGURE 3}

Model-checking versus simulation. Satisfiability of the studied property could be tested by simulation, seeing whether TH3 goes to 0 over time. Nevertheless, two major reasons argue in favor of the model-checking procedure. On the one hand, ECA models infinite timed traces, in contrast to simulation which shows THPN behavior during a finite time, only. Consequently, we cannot be sure that the model reaches a stable state. On the other hand, simulation shows a unique timed trace (conflicts are not explicitly treated), while our model-checking procedure handles timed traces corresponding to all conflict resolutions. Consequently, a conflict resolution could disagree with the property, whereas the simulation trace could agree with it.

\section{Final Decision}

As usual, to extract common traces between the two automata $A_{M}$ and $A_{\neg f}$, we need to perform the product of these automata and to decide whether the set of timed traces of the product automaton, noted $A_{p}$, is empty (emptiness decision). In our case, a specific product integrating quantitative notions is required. We then define a product, called the semantic product and we extended the usual emptiness decision procedure [8] deciding whether the set of traces is empty or not [10]. The semantic product consists in making the conjunction of atoms and of clock constraints. If there is an edge in $A_{M}$ such that $\left(l_{1}, m(T H 4)=0, l_{2}\right.$, True $)$ and if there is an edge in $A_{\neg f}$ such that $\left(l_{1}^{\prime}, m(T H 4) \geq 4, l_{2}^{\prime}, x_{\text {Novvent }}=3\right)$ then $\left(\left(l_{1}, l_{1}^{\prime}\right), m(T H 4)=0 \wedge m(T H 4) \geq\right.$ $\left.4,\left(l_{2}, l_{2}^{\prime}\right), x_{\text {NoEvent }}=3\right)$ is an edge of the product automaton $A_{p}$. Our emptiness decision procedure has then been extended so as to remove all inconsistent edges. Then, the edge given below is removed since there is no possible value of $m(T H 4)$ which can simultaneously satisfy $m(T H 4) \geq 4$ and $m(T H 4)=0$. Finally, if no trace is read on the product automaton, we say that the language of the product automaton is empty.

Biological example Considering the timed traces of $A_{M}$ (Figure 2), it is easy to see that at a moment, the concentration of TH3 reaches zero. When the procedure is extended to all timed traces obtained from all conflict resolutions, the emptiness decision procedure shows that the concentration of TH3 eventually reaches zero. 


\section{Discussion}

From the point of view of our biological example, we show in this paper that when the concurrent actions of D2 and D3 on TH are modeled, the negative loop always overcomes the positive loop. These conclusions are in contradiction with the usual assumption that the intra-cellular concentration of TH3 becomes sufficient to induce apoptosis thanks to D2 [2]. Then new questions arise: "Are, in a same cell, D2 and D3 co-expressed ? If the two enzymes are expressed in the same cells, what is really the action of D2 ? How would evolve a cell whose D2 expression is blocked ?" Since it is difficult to answer these new questions from an in vivo biological model, an in vitro cellular model is nowadays developed by the developmental biologists we work with. Hypotheses formulated from the in silico model will then be tested on the in vitro cellular model before experimentation on the in vivo model. The in vitro cellular model then corresponds to an intermediate model, indispensable for the in silico model exploitation.

From a theoretical point of view, we developed in this paper a model-checking procedure for THPN. The global procedure has been proved to be correct and complete: we proved that the THPN model satisfies the studied property $f$ if and only if the language of the product automaton is empty. This work opens two main research directions. Firstly, THPN correspond to a restricted class of HFPN in which the functional aspect has been removed. We then plan to re-introduce functions by restricting their expressiveness. Secondly, we would like to model check parametric models in which some dynamical parameters are treated symbolically. It would then be possible to answer this king of questions: "What is the speed value of a transition allowing the model to satisfy a given property ?" The presented model-checking procedure seems to be an powerful enough basis to develop these points on the long range.

Acknowledgments We sincerely thank the Laboratory of Evolution and Development and more particularly N. Pollet and R. Thuret.

\section{References}

[1] R. Alur, L. Fix, and T. Henzinger. Event-clock automata: a determinizable class of timed automata. Theor. Computer Science, 211:253-273, 1999. pages

[2] L. Cai and D. Brown. Expression of type 2 iodothyronine deiodinase marks the time that a tissue responds to thyroid hormone-induced metamorphosis in Xenopus laevis. Dev. Biology, 266:87-95, 2003. pages

[3] R. David and H. Alla. Discrete, Continuous, and Hybrid Petri Nets., volume 3-540-22480-7. Springer, 2005. pages 
[4] J.D. Furlow and E.S. Neff. A developmental switch induced by thyroid hormone: Xenopus laevis metamorphosis. TRENDS in Endocrinology and Metabolism, 17:40-47, 2006. pages

[5] D. Germain, R. Schwartzman, W. Croteau, A. Kanamori, Z. Wang, D. Brown, and V Galton. A thyroid hormone-regulated gene in Xenopus laevis encodes a type III iodothyronine 5-deiodinase. Dev. Biology, 91:7767-7771, 1994. pages

[6] H. Huang, N. Marsh-Armstrong, and D. Brown. Metamorphosis is inhibited in transgenic Xenopus laevis tadpoles that overexpress type 3 deiodinase. Dev. Biology, 96:962-967, 1999. pages

[7] H. Matsuno, Y. Tanaka, H. Aoshima, A. Doi, M. Matsui, and S. Miyano. Biopathways representation and simulation on hybrid functional Petri nets. In Silico Biology, 3:389-404, 2003. pages

[8] J-F. Raskin and P-Y. Schobbens. The logic of event clocks. Journal of Automata, Languages and Combinatorics, 4:247-282, 1999. pages

[9] R. Thomas and R. d'Ari. Biological feedback. CRC Press, 1990. pages

[10] S. Troncale, J.-P. Comet, and G. Bernot. Verification of Timed Hybrid Petri Nets with temporal logic. Technical report, IBISC, 2007. pages

[11] S. Troncale, R. Thuret, Ben C., N. Pollet, J.-P. Comet, and G. Bernot. Modelling of the th-dependent regulation of tadpole tail resorption. In RIAMS, 2007. pages

[12] Z. Wang and D. Brown. Thyroid hormone-induced gene expression program for amphibian tail resorption. Journal of Biological Chemistry, 268:16270-16278, 1993. pages

[13] J. Wong and Y.-B. Shi. Coordinated regulation of and transcriptional activation by xenopus thyroid hormone and retinoid X receptors. Journal of Biological Chemistry, 270:18479-18483, 1995. pages

Fig. 1. THPN of competitive regulation between D2 and D3 and its evolution graph.

Fig. 2. Event Clock automaton $A_{M}$. To simplify, atoms labeling edges only consider marking of places TH3 and TH4 and speed values of the continuous transitions $T_{3}$ and $T_{4}$.

Fig. 3. Event Clock automaton $A_{\neg f}$. 


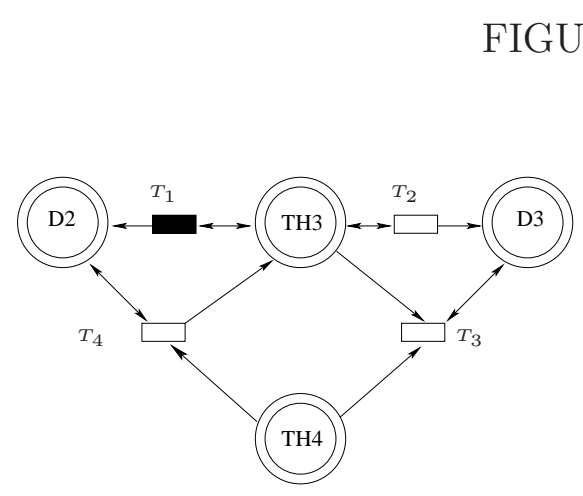

THPN model

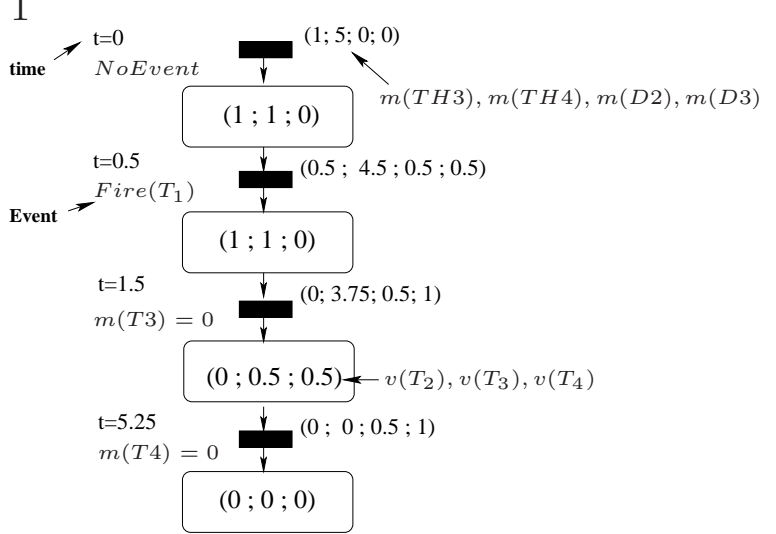

Evolution graph

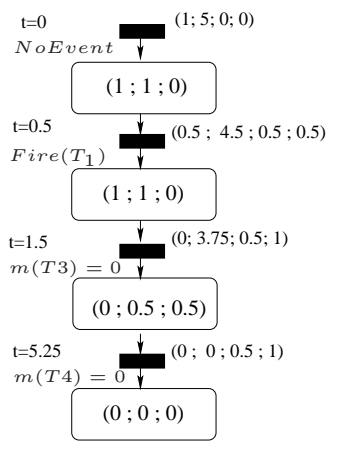

FIGURE 2

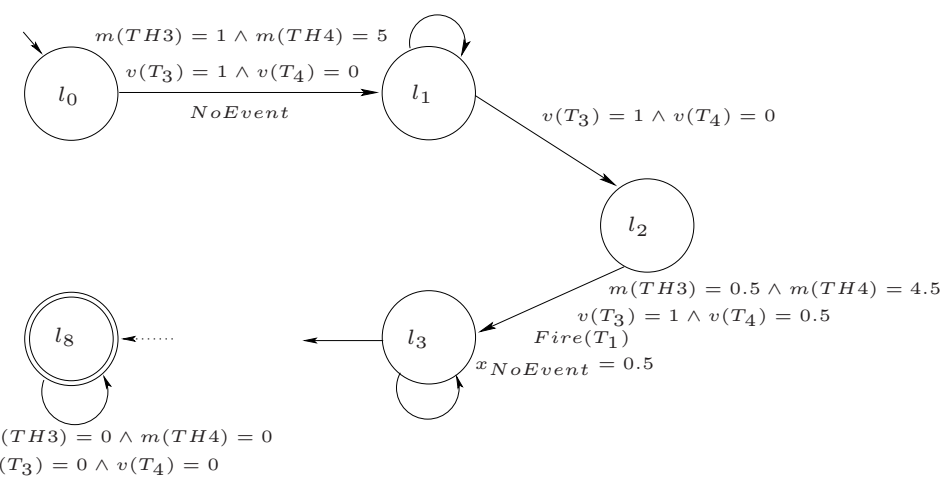

FIGURE 3

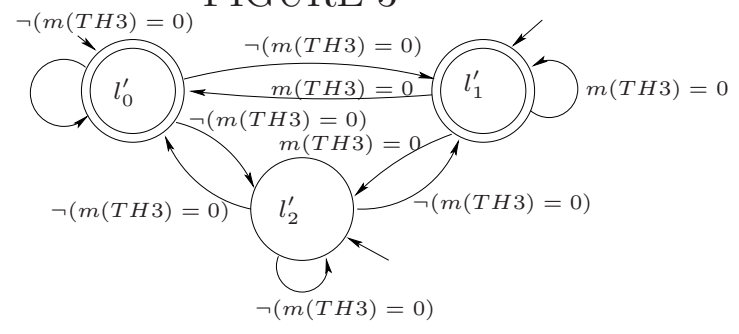

